\title{
Hessian Fly (Mayetiola destructor) Attack Causes a Dramatic Shift in Carbon and Nitrogen Metabolism in Wheat
}

\author{
Lieceng Zhu, ${ }^{1}$ Xuming Liu, ${ }^{1}$ Xiang Liu, ${ }^{1}$ Richard Jeannotte, ${ }^{2}$ John C. Reese, ${ }^{1}$ Marion Harris, ${ }^{3}$ \\ Jeffrey J. Stuart, ${ }^{4}$ and Ming-Shun Chen ${ }^{1}$ \\ ${ }^{1}$ Department of Entomology, ${ }^{2}$ Division of Biology, Kansas State University, Manhattan, KS 66506, U.S.A.; ${ }^{3}$ Department \\ of Entomology, North Dakota State University, Fargo, ND 58105, U.S.A.; ${ }^{4}$ Department of Entomology, Purdue University, \\ West Lafayette, IN 47907, U.S.A.
}

Submitted 15 August 2007. Accepted 18 September 2007.

Carbon and nitrogen $(\mathrm{C} / \mathrm{N})$ metabolism and allocation within the plant have important implications for plantparasite interactions. Many plant parasites manipulate the host by inducing $\mathrm{C} / \mathrm{N}$ changes that benefit their own survival and growth. Plant resistance can prevent this parasite manipulation. We used the wheat-Hessian fly (Mayetiola destructor) system to analyze $\mathrm{C} / \mathrm{N}$ changes in plants during compatible and incompatible interactions. The Hessian fly is an insect but shares many features with plant pathogens, being sessile during feeding stages and having avirulence (Avr) genes that match plant resistance genes in gene-forgene relationships. Many wheat genes involved in $\mathrm{C} / \mathrm{N}$ metabolism were differentially regulated in plants during compatible and incompatible interactions. In plants during compatible interactions, the content of free carbon-containing compounds decreased $36 \%$, whereas the content of free nitrogen-containing compounds increased $46 \%$. This $\mathrm{C} / \mathrm{N}$ shift was likely achieved through a coordinated regulation of genes in a number of central metabolic pathways, including glycolysis, the tricarboxylic acid cycle, and amino-acid synthesis. Our data on plants during compatible interactions support recent findings that Hessian fly larvae create nutritive cells at feeding (attack) sites and manipulate host plants to enhance their own survival and growth. In plants during incompatible interactions, most of the metabolic genes examined were not affected or downregulated.

For many plant parasites, attack of the susceptible host plant is associated with the creation of a zone of "metabolic habitat modification" (Goethals et al. 2001). In this zone, the parasite experiences a selective advantage because of enhanced nutrition and reduced plant defense. The best-known example of this is the soil pathogen Agrobacterium tumeficiens, which creates a tumorous growth of plant tissues in the stem (i.e., crown gall disease) and then benefits from the tumor's predominant

Corresponding author: Ming-Shun Chen; USDA-ARS and Department of Entomology, 123 Waters Hall; Telephone: +1.785.532.4719; Fax: +1.785.532.6232; E-mail: mchen@ksu.edu

Mention of commercial or proprietary product does not constitute endorsement by the United States Department of Agriculture.

* The $\boldsymbol{e}$-Xtra logo stands for "electronic extra" and indicates a supplemental table is published online. carbon-nitrogen source, the unusual amino acid-like opines that are specifically catabolized by certain Agrobacterium strains (Zupan et al. 2000). Many types of plant parasites manipulate susceptible hosts, including fungi, bacteria, nematodes, mites, and insects (Agrios 1997; Rohfritsch 2005). An important aspect of parasite manipulation is a change in carbon and nitrogen metabolism and allocation within the plant. The parasite attack site becomes a sink and, therefore, competes with other plant tissues for photoassimilates (Stone and Schönrogge 2003).

Many plants defend themselves against parasite manipulation via gene-for-gene resistance (Agrios 1997). This requires three important steps, surveillance, signal transduction, and the production of defense chemicals (Dangl and McDowell 2006; Ferry et al. 2004; Kessler and Baldwin 2002; Walling 2000). In the first step, the plant's surveillance system detects parasite attack. The detected signal is then transduced through a network of signal transduction pathways, which eventually leads to the production of defense chemicals (Hahlbrock et al. 2003; Wan et al. 2002). In gene-for-gene resistance, the surveillance system consists of the product of a resistance $(R)$ gene. The majority of $R$ genes currently characterized encode $\mathrm{R}$ proteins with leucine-rich repeats and a nucleotide-binding site (Kaloshian 2004; Meyers et al. 1999; Nimchuk et al. 2003). During signal recognition, the $\mathrm{R}$ product interacts with the product of the parasite's matching avirulence $(A v r)$ gene either directly or indirectly (Dangl and McDowell 2006; Dodds et al. 2006; Tang et al. 1996). The defense that results from the RAvr interaction presumably prevents the parasite from manipulating carbon and nitrogen $(\mathrm{C} / \mathrm{N})$ metabolism and allocation within the plant.

We studied $\mathrm{C} / \mathrm{N}$ metabolism and allocation of a wheat genotype, 'Molly', under attack by a virulent and an avirulent biotype Hessian fly (Mayetiola destructor). The Hessian fly is a member of a family of insects, the Cecidomyiidae (Order: Diptera), whose plant-feeding members are commonly referred to as gall midges. Many, if not most, gall midges manipulate the susceptible host by creating a tissue of nutritive cells at the attack site (Rohfritsch 2005). Frequently, but not always, the nutritive tissue is surrounded or covered by an outgrowth of tissue, i.e., the macroscopic structure referred to as a covering gall. The Hessian fly creates a nutritive tissue 1 to 2 days after initially attacking the abaxial epidermal cells of the leaf sheath of wheat seedlings but does not produce a covering gall (Harris et al. 2006). Soon after the nutritive cells appear, the contents of the nutritive cells start breaking down and, probably as a result of larval sucking, move through compromised cell walls 
towards the larva. The larva grows rapidly, completing its development in 10 to 12 days (Gagne and Hatchet 1989). The Hessian fly-induced nutritive tissue has a serious impact on the wheat seedling. Leaves younger than the attacked leaf fail to develop beyond the initial epidermal layers (Harris et al. 2006) and do not elongate (Anderson and Harris 2006). Unless one or more tillers escape larval manipulation, the plant dies without producing seed during compatible interactions. Because of this, the Hessian fly is a serious pest of wheat in North America and North Africa (Berzonsky et al. 2003).

$R$ gene-mediated resistance confers complete resistance to Hessian fly attack (Berzonsky et al. 2003; Harris et al. 2003). When a Hessian fly larva attacks a plant with an effective $R$ gene, it manipulates the growth of the attacked leaf sheath during the next two days (Anderson and Harris 2006). However, the larva typically is dead 3 to 5 days after its initial attack and does not grow before dying, i.e., it does not obtain nutrients from the plant (Shukle et al. 1990). As the resistant plant grows, it compensates for early larva-induced growth deficits and suffers no significant fitness cost (K. G. Anderson and M. O. Harris, unpublished data). Thus, 3 days into the plant-parasite interaction, the susceptible plant has been manipulated to create a nutritive tissue that benefits the Hessian fly larva and harms the plant. In contrast, the resistant plant has defended itself successfully, presumably by preventing the early phases of parasite manipulation (Anderson and Harris 2006; Harris et al. 2006). Classical Mendelian genetic studies have shown gene-for-gene interactions between grass $R$ genes and Hessian fly $A v r$ genes (Harris et al. 2003). The Hessian fly can evolve virulence to a particular $R$ gene, and the 'defeated' $R$ gene is no longer useful for resistance in agriculture.

To study the molecular processes involved in wheat-Hessian fly interaction, we used microarrays to identify genes that were differently regulated in compatible and incompatible interactions. Our microarray data revealed a significant alteration in the levels of transcripts of genes involved in $\mathrm{C} / \mathrm{N}$ metabolism (M.-S. Chen, unpublished results). Since $\mathrm{C} / \mathrm{N}$ metabolism and allocation have important implications in plant-parasite interactions (Girousse et al. 2005; Schwachtje et al. 2006), we ex- amined the changes in transcript levels of several genes involved in central metabolic pathways, including glycolysis, the tricarboxylic acid (TCA) cycle, the pentose phosphate pathway (PPP), and amino-acid synthesis. Larval attack and plant susceptibility or resistance had major impacts on the regulation of metabolic genes. Furthermore, we analyzed the accumulation of $\mathrm{C} / \mathrm{N}$-containing compounds (C-compounds and $\mathrm{N}$-compounds, respectively) at the larva-attack site using metabolic profiling. Combined analysis of transcript changes and metabolic profiling data indicated that, at the larva-attack site, there were differences in primary metabolite levels when compatible and incompatible interactions were compared. The elevation of selected N-compounds at the attack site during compatible interactions was noted and corresponding changes at the RNA levels for several genes involved in glycolysis, TCA cycle, PPP, and amino-acid synthesis pathways were correlated with these changes. In contrast, during incompatible interactions, metabolic gene RNA levels were either not altered or were down-regulated.

\section{RESULTS}

Dramatic shift from C-compounds to $\mathbf{N}$-compounds.

A wheat cultivar containing the $R$ gene $H 13$ ('Molly'; Liu et al. 2005) and two Hessian fly biotypes, $G P$ (avirulent, larvae die on $H 13$ plants) and $v H 13$ (virulent, larvae survive on $H 13$ plants), were used to create plant-insect treatments. Seedlings without infestation were used as control plants, seedlings infested with $G P$ were defined as plants during incompatible interactions, while seedlings infested with $v H 13$ were defined as plants during compatible interactions. Samples were collected 3 days after larvae started attack at the base of the plant. The metabolic profiling by way of measuring free metabolites was conducted at the Metabolic Core Facility at the University of California at Davis. More than 400 compounds can be analyzed at this facility (Table S1). In our assays, 82 unique metabolites were reproducibly detected. The majority (60) of these metabolites were C-compounds, including 19 sugars, 10 sugar alcohols, 21 organic acids, and 10 fatty acids or lipids

Table 1. Summary of detected metabolites ${ }^{\mathrm{a}}$

\begin{tabular}{|c|c|c|c|c|c|c|}
\hline \multirow[b]{2}{*}{ Metabolites } & \multirow[b]{2}{*}{ No. } & \multicolumn{3}{|c|}{ Total concentration in each class (\% of total peak area times 100 ) } & \multicolumn{2}{|c|}{ Percentage changes } \\
\hline & & C & $\mathbf{S}$ & $\mathbf{R}$ & $\mathbf{S}$ & $\mathbf{R}$ \\
\hline \multicolumn{7}{|l|}{ Carbon-containing compounds } \\
\hline Sugars ${ }^{\mathrm{b}}$ & 19 & $1,687.40 \pm 56.7(50.4)$ & $1,325.44 \pm 186.5(62.0)$ & $1,288.09 \pm 87.5(50.4)$ & -21 & -24 \\
\hline Sugar alcohols ${ }^{\mathrm{c}}$ & 10 & $93.44 \pm 5.8(2.8)$ & $64.07 \pm 7.6(3.0)$ & $97.71 \pm 5.0(3.8)$ & -31 & +5 \\
\hline Organic acid ${ }^{\mathrm{d}}$ & 21 & $1.118 .47 \pm 73.7(33.4)$ & $457.28 \pm 34.0(21.4)$ & $785.46 \pm 33.9(30.8)$ & -59 & -30 \\
\hline Fatty acids and lipids ${ }^{\mathrm{e}}$ & 10 & $447.53 \pm 45(13.4)$ & $290.49 \pm 32.1(13.6)$ & $382.25 \pm 32.5(15.0)$ & -35 & -15 \\
\hline Total & 60 & $3,346.85 \pm 139.0(100)$ & $2137.28 \pm 172.5(100)$ & $2,553.51 \pm 134(100)$ & -36 & -24 \\
\hline \multicolumn{7}{|c|}{ Nitrogen-containing compounds } \\
\hline Protein amino acids ${ }^{\mathrm{f}}$ & 14 & $3,256.44 \pm 86.6(73.0)$ & $4,944.52 \pm 277.0(76.3)$ & $4,197.29 \pm 90.5(75.3)$ & 52 & 29 \\
\hline Nonprotein amino acids $\mathrm{g}$ & 5 & $1,121.41 \pm 186.8$ & $1,426.80 \pm 269.7(22.0)$ & $1,307.36 \pm 118.0$ & 27 & 17 \\
\hline Amines ${ }^{\mathrm{h}}$ & 3 & $70.42 \pm 7.25(1.6)$ & $105.48 \pm 2.5(1.6)$ & $66.31 \pm 10.3(1.2)$ & 50 & -6 \\
\hline Total & 22 & $4,448.25 \pm 268.4(100)$ & $6,476.80 \pm 988.4(100)$ & $5,570.95 \pm 140.3(100)$ & 46 & 25 \\
\hline
\end{tabular}

${ }^{\mathrm{a}}$ In control plants $(\mathrm{C})$ and plants during compatible $(\mathrm{S})$ and incompatible $(\mathrm{R})$ interactions. No. $=$ Numbers of metabolites in each class $(P \leq 0.05)$.

${ }^{\mathrm{b}}$ Sugars: ribulose-5-phosphate, glucose, mannose, glucose-6-phosphate, trehalose, inulobiose, fructose, xylose, fructose-6-phosphate, galactose-6-phosphate, sucrose, arabinose, glucose 1 phosphate, ribose, galactose, levanbiose, fucose + rhamnose maltose, and inulotriose.

c Sugar alcohols: myo-inositol, galactinol, glycerolphosphate alpha, mannitol, glycerol-2-phosphate, xylitol, sorbitol, methanolphosphate, inositolmonophosphate, and arabitol.

${ }^{\mathrm{d}}$ Organic acids: gluconic acid, citramalate, quinic acid, alpha ketoglutaric acid, ascorbic acid, fumarate, shikimic acid, malate, threonic acid, aconitic acid, citric acid, glyceric acid, dehydroascorbate, allantoin, ribonic acid, hexonic acid, glycolic acid, lactic acid, pentonic acid, erythronic acid, and xylonic acid.

${ }^{\mathrm{e}}$ Fatty acids and lipids: stearic acid, cis-2-octadecenoic acid, palmitic acid, linolenic acid, linoleic acid, monopalmitin-1-glyceride Isofucostanol, phosphoethanolamine, tocopherol, and 4-methylcholesterol.

${ }^{f}$ Protein amino acids: proline, arginine + ornithine, serine, asparagines, threonine, aspartic acid, isoleucine, valine, alanine, lysine, tyrosine, methionine, glutamic acid, and glycine.

${ }^{g}$ Nonprotein amino acids: citrulline, pipecolic acid, oxoproline, beta alanine, and $\gamma$-amino-n-butyric acid.

${ }^{\text {h }}$ Amines: putrescine, $N$-acetyl-D-mannosamine, and spermidine. 
(Table 1). The remaining metabolites (22) were N-compounds, including 14 protein amino acids, five nonprotein amino acids, and three amines.

There was a dramatic shift in the accumulation of different metabolites in plants during compatible and incompatible interactions following Hessian fly attack. In control plants, $50.4 \%$ of C-compounds were sugars. The other C-compounds were organic acids $(33.4 \%)$, fatty acids or lipids $(13.4 \%)$, and sugar alcohols $(2.8 \%)$. For N-compounds in control plants, $73 \%$ were protein amino acids, $25.2 \%$ were nonprotein amino acids, and $1.6 \%$ were amines (Table 1). Three days after Hessian fly attack was initiated, plants during compatible interactions had a $36 \%$ reduction in the relative concentration of total C-compounds. Specifically, there was a $21 \%$ decrease in sugars, a $31 \%$ decrease in sugar alcohols, a $59 \%$ decrease in organic acids, and a $35 \%$ decrease in fatty acids or lipids. In contrast, the same plants had a $46 \%$ increase in the relative concentration of $\mathrm{N}$-compounds, including a $52 \%$ increase in protein amino acids, a $50 \%$ increase in amines, and a $27 \%$ increase in nonprotein amino acids. The combination of a decrease in $\mathrm{C}$ compounds and an increase in $\mathrm{N}$-compounds resulted in a $\mathrm{C} / \mathrm{N}$ ratio of 0.33 , less than one-half of the $\mathrm{C} / \mathrm{N}$ ratio $(0.75)$ observed in control plants. The metabolic shift induced by Hessian fly attack was less dramatic and less consistent in plants during incompatible interactions. Plants during incompatible interactions had a $24 \%$ reduction in the total concentration of $\mathrm{C}$-compounds and a $25 \%$ increase in the total concentration of $\mathrm{N}$-compounds, resulting in a $\mathrm{C} / \mathrm{N}$ ratio of 0.46 .

Principal component analysis was conducted on the 82 detected metabolites to provide an overall evaluation of metabolic profiles of the three plant treatments. The first two components, which explained $54 \%$ of the variance, were used to construct score plots as indicated in Figure 1. In the score plot, three treatments formed three distinctive groups, demonstrating that metabolic phenotypes were distinctively different between control plants, plants during compatible interactions, and plants during incompatible interactions. Moreover, the distance between control plants and plants during compatible interactions was greater than that between control plants and plants during incompatible interactions, again indicating that the alteration of metabolic profiles in wheat caused by Hessian fly attack was greater in plants during compatible interactions than in plants during incompatible interactions. We also noted that despite the overall reduction of the $\mathrm{C} / \mathrm{N}$ ratio following Hessian fly attack, the changes in concentration of specific metabolites were very different within each metabolic group (Table 2).

\section{Differential regulation of key metabolic pathways.}

To determine the mechanism for such a dramatic shift from $\mathrm{C}$-compounds to $\mathrm{N}$-compounds, we examined the changes in the levels of gene transcripts involved in related metabolic pathways in the three plant treatments described above. Since the glycolysis, the TCA cycle, and the PPP are central pathways for energy metabolism and for generating intermediates for biosynthesis, we examined the RNA levels for representative genes in these pathways using Northern blots. In addition, the changes in the levels of transcripts of several genes involved in amino-acid synthesis were also determined.

\section{Glycolysis.}

Glycolysis is the pathway for degradation of sugars and other carbohydrates. Ten major genes are involved in plant glycolysis. The first three genes (hexokinase, phosphoglucose isomerase, and phosphofructokinase) were not analyzed because of a lack of probes. The other seven genes, encoding fructose 1,6-biphosphate aldolase, triose phosphate isomerase, glyceraldehyde-3-phosphate dehydrogenase, phosphoglyerate kinase, phosphoglycero-mutase, enolase, and pyruvate kinase, were analyzed via Northern blots. All seven genes had similar transcript patterns following Hessian fly attack. In plants during compatible interactions, the transcript levels of six of the seven genes increased slightly from 24 to $48 \mathrm{~h}$, the transcript levels of all the seven genes increased strongly at $72 \mathrm{~h}$ and remained high afterwards (Fig. 2A). In plants during incompatible interactions, the transcript levels of these genes either decreased at 24 and $48 \mathrm{~h}$ and then recovered to normal levels afterward or did not change during the whole experimental period.

\section{The TCA cycle and other related enzymes.}

The TCA cycle is the central pathway in metabolism. It serves as the final step for breakdown of carbohydrates, fatty

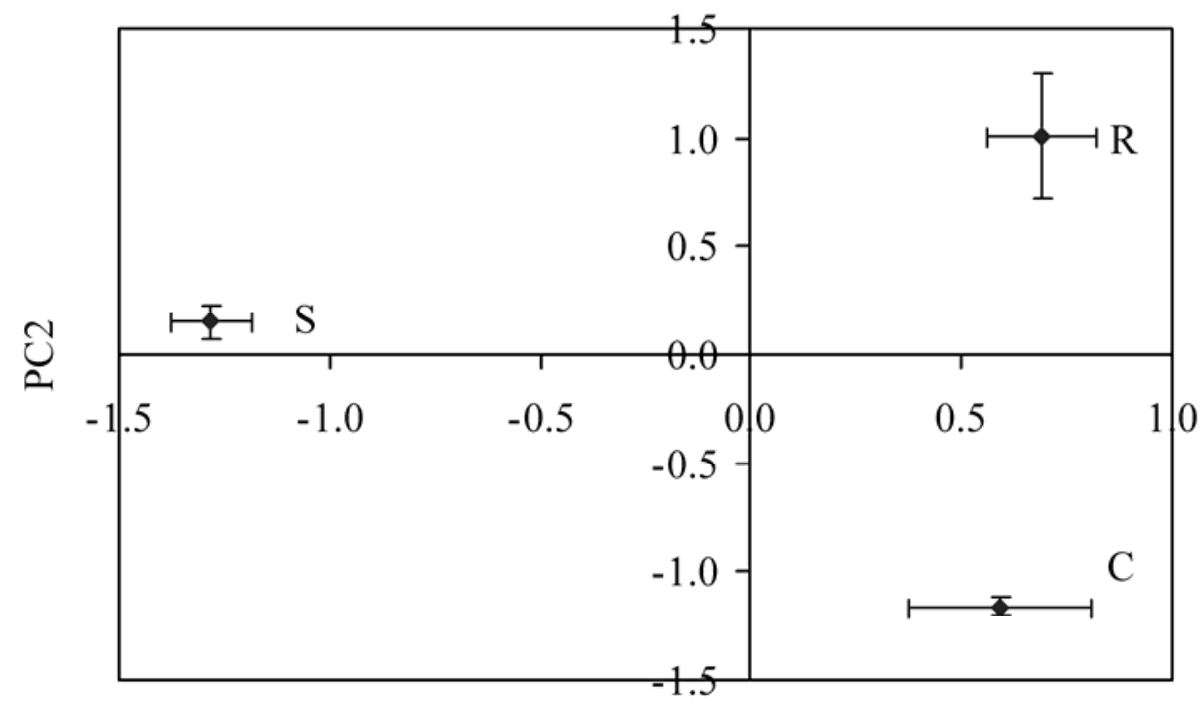

PC1

Fig. 1. Principle component analysis (PCA) of the metabolic profiles of 82 metabolites in control plants (C), plants during compatible interactions (S), and plants during incompatible interactions (R). The first two principle components, explaining $54 \%$ of the variance, were used for plotting the scores. The principle component 1 (PC1) accounts for $34 \%$ of variance; principle component 2 (PC2) accounts for $20 \%$ of the variance. 
acids, or amino acids to produce energy but may also use carbohydrates, fatty acids, or amino acids to produce intermediates for the biosynthesis of various molecules. There are eight enzymes directly involved in the TCA cycle and many other related enzymes. However, because of a lack of probes, only six genes were examined. In contrast to the coordinated regulation of all genes in glycolysis, the genes encoding TCA cyclerelated enzymes appeared to have different responses to Hessian fly attack. Like the glycolysis genes, TCA genes encoding malate dehydrogenase, isocitrate dehydrogenase, succinate dehydrogenase, and $\mathrm{NAD}^{+}$-malic enzyme exhibited similar transcript patterns to those of the genes in glycolysis. Namely, the transcript levels of these genes increased in plants during compatible interactions, but they either decreased slightly or did not change in plants during incompatible interactions (Fig. 2B). However, the transcripts of the other two genes, $\alpha$-ketoglutarate dehydrogenase (2-oxoglutarate dehydrogenase, E1 subunit) and phosphoenolpyruvate carboxylase, exhibited a different pattern. The levels of their transcripts did not change significantly in plants during either incompatible or compatible interactions. The unbalanced increase in the transcript levels of some of the TCA genes might indicate that the TCA cycle was regulated for the production of specific intermediates for biosynthesis in plants during compatible interactions.

\section{The PPP.}

PPP is a pathway that produces NADPH, a common donor of hydrogen and electrons in reductive biosynthesis, such as amino-acid synthesis. Unfortunately, probes were not available for the genes involved in this pathway, except for glucose-6phosphte dehydrogenase (G6PD), the first and, also, rate-limiting step in PPP. The level of G6PD transcripts increased significantly in plants during compatible interactions but did not change in plants during incompatible interactions, although the increase of G6PD transcripts in plants during compatible interactions appeared delayed in comparison with the genes in glycolysis.

\section{Amino-acid synthesis.}

The increase in the concentration of amino acids in wheat plants following Hessian fly attack may be caused by one or a combination of the following mechanisms: i) increased aminoacid synthesis, ii) protein degradation, or iii) decreased aminoacid catabolism. To distinguish between these possibilities, the transcript levels of the genes encoding glutamine synthetase (Gln-S), glutamate synthase (Glu-S), pyrroline-5-carboxylate synthetase (P5CS) (in the pathway for proline synthesis), and methionine synthase (Met-S) were analyzed in plants during compatible and incompatible interactions. The transcript levels

Table 2. Relative concentration of each metabolite ${ }^{\mathrm{a}}$ and percentage changes of concentration ${ }^{\mathrm{b}}$

\begin{tabular}{|c|c|c|c|c|c|}
\hline \multirow[b]{2}{*}{ Name } & \multicolumn{3}{|c|}{ Means of relative concentration } & \multicolumn{2}{|c|}{ Percentage change } \\
\hline & $\mathbf{C}$ & $\mathbf{S}$ & $\mathbf{R}$ & $\mathbf{S}$ & $\mathbf{R}$ \\
\hline \multicolumn{6}{|l|}{ Sugars and sugar alcohols } \\
\hline Glucose & $583.52 \pm 53.34$ & $172.03 \pm 17.51$ & $475.73 \pm 47.80$ & -71 & $\mathrm{~N}$ \\
\hline Glucose-6-phosphate & $2.31 \pm 0.19$ & $0.97 \pm 0.08$ & $1.97 \pm 0.15$ & -58 & $\mathrm{~N}$ \\
\hline Fructose & & & & -49 & $\mathrm{~N}$ \\
\hline Mannose & $7.29 \pm 0.99$ & $2.81 \pm 0.40$ & $7.69 \pm 0.85$ & -61 & $\mathrm{~N}$ \\
\hline Trehalose & $17.14 \pm 1.32$ & $7.94 \pm 0.90$ & $10.94 \pm 1.23$ & -54 & -36 \\
\hline Galactose & $2.47 \pm 0.48$ & $2.81 \pm 0.36$ & $15.57 \pm 7.46$ & $\mathrm{~N}$ & 530 \\
\hline Ribulose-5-phosphate & $40.00 \pm 3.05$ & $5.84 \pm 1.44$ & $40.24 \pm 3.71$ & -85 & $\mathrm{~N}$ \\
\hline Xylose & $2.84 \pm 0.42$ & $1.45 \pm 0.20$ & $3.26 \pm 0.43$ & -49 & $\mathrm{~N}$ \\
\hline Inulotriose & $198.51 \pm 17.21$ & $623.11 \pm 157.34$ & $131.54 \pm 22.63$ & 214 & $\mathrm{~N}$ \\
\hline Myo-inositol & $28.57 \pm 2.75$ & $10.93 \pm 0.97$ & $20.88 \pm 1.38$ & -64 & $\mathrm{~N}$ \\
\hline Galactinol & $5.06 \pm 0.35$ & $3.59 \pm 0.25$ & $15.24 \pm 1.69$ & $\mathrm{~N}$ & 201 \\
\hline Arabitol & $0.64 \pm 0.11$ & $1.74 \pm 0.15$ & $3.28 \pm 0.46$ & 174 & 416 \\
\hline \multicolumn{6}{|l|}{ Organic acids } \\
\hline Citric acid & $68.17 \pm 4.69$ & $34.50 \pm 6.14$ & $70.32 \pm 9.33$ & -49 & $\mathrm{~N}$ \\
\hline$\alpha$-Ketoglutaric acid & $19.00 \pm 1.39$ & $5.21 \pm 0.76$ & $5.95 \pm 0.50$ & -73 & -69 \\
\hline Fumarate & $23.71 \pm 1.80$ & $8.90 \pm 0.82$ & $20.52 \pm 1.26$ & -62 & $\mathrm{~N}$ \\
\hline Malate & $398.27 \pm 26.77$ & $154.10 \pm 25.70$ & $212.06 \pm 31.97$ & -61 & -47 \\
\hline Ascorbic acid & $15.60 \pm 3.21$ & $4.53 \pm 0.30$ & $2.45 \pm 0.35$ & -71 & -84 \\
\hline Dehydroascorbate & $34.58 \pm 1.07$ & $17.95 \pm 2.01$ & $0.00 \pm 0.00$ & -48 & -100 \\
\hline Threonic acid & $13.11 \pm 1.26$ & $6.00 \pm 0.50$ & $29.24 \pm 2.54$ & -54 & 123 \\
\hline Glyceric acid & $13.44 \pm 1.38$ & $6.93 \pm 0.92$ & $27.15 \pm 2.19$ & -48 & 102 \\
\hline Gluconic acid & $1.50 \pm 0.16$ & $0.10 \pm 0.01$ & $3.89 \pm 0.22$ & -93 & 160 \\
\hline Citramalate & $1.28 \pm 0.17$ & $0.17 \pm 0.11$ & $1.54 \pm 0.06$ & -87 & $\mathrm{~N}$ \\
\hline Shikimic acid & $110.4 \pm 9.01$ & $42.9 \pm 7.08$ & $59.4 \pm 5.58$ & -61 & -46 \\
\hline Quinic acid & $267.40 \pm 22.07$ & $68.67 \pm 6.75$ & $212.38 \pm 15.14$ & -74 & $\mathrm{~N}$ \\
\hline \multicolumn{6}{|l|}{ Protein amino aicds } \\
\hline Proline & $90.60 \pm 3.19$ & $1,222.20 \pm 82.08$ & $60.40 \pm 6.13$ & 1249 & $\mathrm{~N}$ \\
\hline Arginine + ornithine & $7.04 \pm 0.70$ & $11.50 \pm 1.26$ & $6.90 \pm 0.85$ & 64 & $\mathrm{~N}$ \\
\hline Serine & $151.70 \pm 14.40$ & $243.00 \pm 27.61$ & $135.30 \pm 12.12$ & 60 & $\mathrm{~N}$ \\
\hline Asparagine & $364.00 \pm 40.79$ & $582.60 \pm 8.03$ & $929.80 \pm 115.37$ & $\mathrm{~N}$ & 155 \\
\hline Threonine & $24.80 \pm 2.72$ & $36.20 \pm 3.76$ & $25.80 \pm 3.76$ & 46 & $\mathrm{~N}$ \\
\hline Aspartic acid & $420.30 \pm 22.81$ & $584.90 \pm 15.42$ & $293.80 \pm 19.08$ & 39 & $\mathrm{~N}$ \\
\hline Alanine & $1,087.10 \pm 31.26$ & $1,242.50 \pm 53.66$ & $1,584.60 \pm 90.62$ & $\mathrm{~N}$ & 46 \\
\hline Tyrosine & $34.10 \pm 2.15$ & $30.70 \pm 2.50$ & $48.20 \pm 5.01$ & $\mathrm{~N}$ & 42 \\
\hline Glycine & $210.30 \pm 13.33$ & $140.60 \pm 3.70$ & $333.20 \pm 35.71$ & -33 & 58 \\
\hline \multicolumn{6}{|c|}{ Nonprotein amino acids and amines } \\
\hline Citrulline & $0.01 \pm 0.01$ & $0.64 \pm 0.19$ & $0.070 \pm 0.04$ & 5137 & $\mathrm{~N}$ \\
\hline Pipecolic acid & $7.61 \pm 0.72$ & $11.88 \pm 0.99$ & $101.45 \pm 16.10$ & $\mathrm{~N}$ & 1234 \\
\hline Putrescine & $52.73 \pm 6.18$ & $92.41 \pm 8.53$ & $50.13 \pm 7.87$ & 75 & $\mathrm{~N}$ \\
\hline
\end{tabular}


of Glu-S, P5CS, and Met-S all increased in plants during compatible interactions, but they either decreased or did not change in plants during incompatible interactions (Fig. 2D). On the other hand, the transcript pattern of Gln-S was distinct. At earlier timepoints (12 and $24 \mathrm{~h}$ after infestation), the transcript level of Gln-S increased considerably in plants during incompatible interactions but did not change in plants during compatible interactions. Interestingly, at later timepoints ( $72 \mathrm{~h}$ and after), the transcript level of Gln-S increased in plants during compatible interactions but did not change in plants during incompatible interactions. Given Gln-S is one of the most important enzymes in nitrogen assimilation, the timing of Gln-S upregulation in plants during incompatible and compatible interactions may reflect difference in the timing of $\mathrm{N}$-compound accumulation.

\section{Perturbation in gene regulation localized.}

In order to understand if the impact of Hessian fly attack is localized or systemic, we examined the regulation of a few

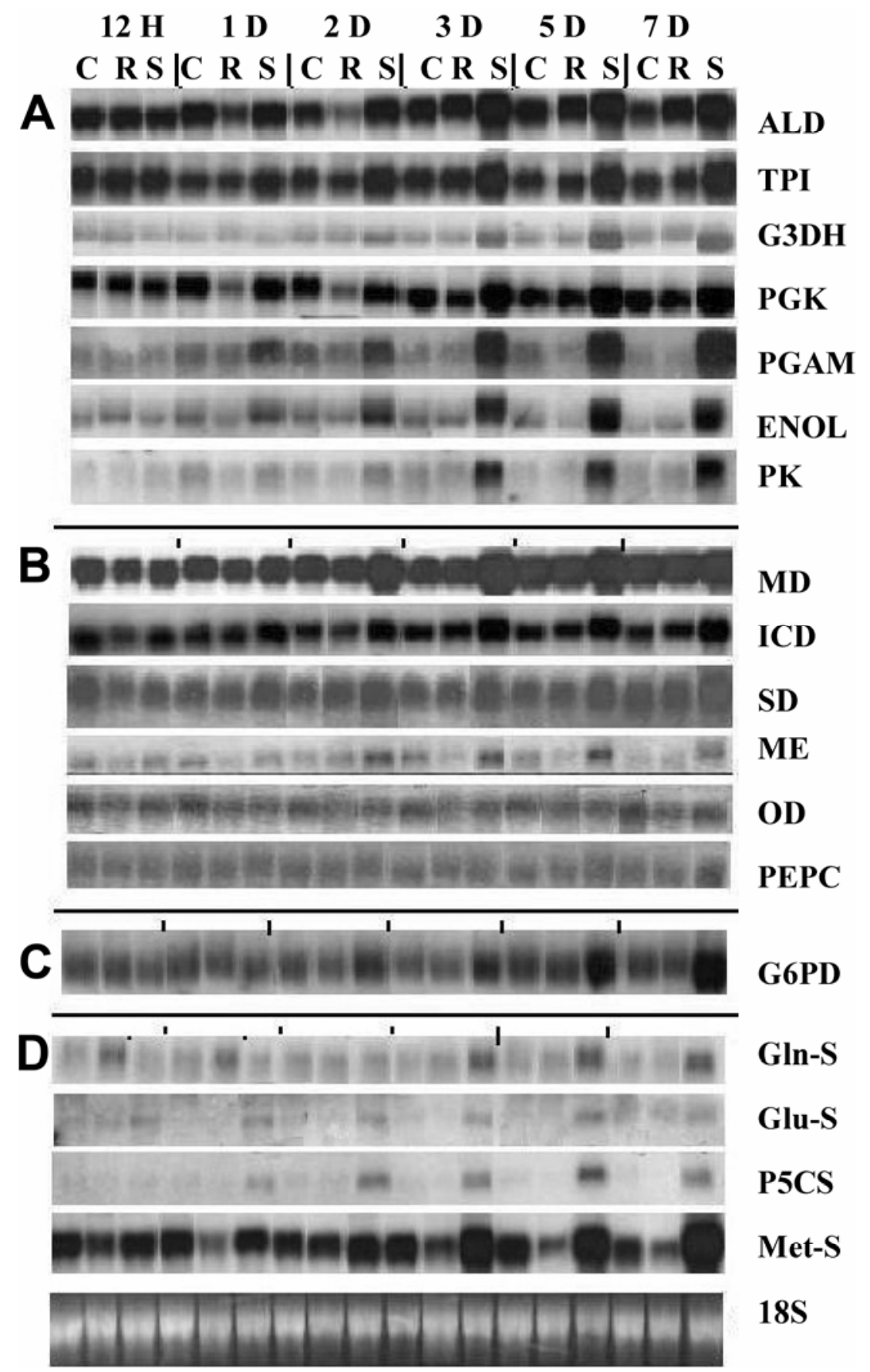

Fig. 2. Northern blot analysis of genes involved in glycolysis, tricarboxylic acid cycle (TCA), pentose phosphate pathway (PPP), and amino acid synthesis pathways 12, 24 (1 day), 48 (2 days), 72(3 days), 120 (5 days), and 168 (7 days) h (12 H, 1 D, 2 D, 3D, 5 D, and 7 D, respectively) after Hessian fly attack. $\mathrm{C}, \mathrm{R}$, and $\mathrm{S}=$ control plants, plants during incompatible interactions, and plants compatible interactions, respectively. A, Transcript analysis of genes involved in glycolysis. ALD = fructose 1,6-biphosphate aldolase,TPI $=$ triose phosphate isomerase, G3DH $=$ glyceraldehyde-3-phosphate dehydrogenase, PGK $=$ phosphoglyerate kinase, $\mathrm{PGAM}=$ phosphoglycero-mutase, $\mathrm{ENOL}=$ enolase, and $\mathrm{PK}=$ pyruvate kinase . B, Transcript analysis of genes involved in TCA. $\mathrm{MD}=$ malate dehydrogenase, $\mathrm{ICD}=$ isocitrate dehydrogenase, $\mathrm{SD}=$ succinate dehydrogenase, $\mathrm{ME}=\mathrm{NAD}{ }^{+}$-malic enzyme, $\mathrm{OD}=\alpha$-ketoglutarate dehydrogenase (2-oxoglutarate dehydrogenase, E1 subunit), and PEPC = phosphoenolpyruvate carboxylase. C, Transcript analysis of a gene encoding glucose6-phosphate dehydrogenase (G6PD), which is involved in the PPP. D, Transcript analysis of genes involved in amino acid synthesis. Gln-S = glutamine synthetase, Glu-S = glutamate synthase, P5CS = pyrroline-5-carboxylate synthetase, Met-S = methionine sythase, and $18 \mathrm{~S}=18 \mathrm{~S}$ ribosomal RNA. 
representative genes in five different locations of the three types of $H 13$ plants, control plants (without infestation), plants during incompatible interactions (infested with the avirulent biotype $G P$ ), and plants during compatible interactions (infested with the virulent biotype vH13). Our results demonstrated the impact of Hessian fly attack on gene regulation was limited to the attack (feeding) site and immediately above the feeding site (Fig. 3). Under our experimental conditions, all genes exhibited similar regulation patterns. The levels of their transcripts increased at the feeding site and immediately above the feeding site but did not change in the second leaf blade (the feeding site was on the second leaf sheath), in the third leaf sheath or in the first leaf sheath in plants during compatible interactions. Similarly, these genes were slightly down-regulated at and immediately above the feeding site but not affected in other locations in plants during incompatible interactions. These observations indicated that the perturbation of Hessian fly attack on the regulation of metabolic genes was localized during both compatible and incompatible interactions at $72 \mathrm{~h}$.

\section{DISCUSSION}

\section{Unique regulation of primary metabolic wheat genes} in response to Hessian fly attack.

In plant-pathogen interactions, much of the difference in gene regulation between plants during compatible interactions and plants during incompatible interactions is quantitative (Maleck et al. 2000; Tao et al. 2003). Typically, changes in the levels of gene transcripts induced by pathogen attack occur more quickly and with greater magnitude in plants during incompatible interactions than in plants during compatible interactions, but the direction (either up- or downregulation) of the alteration is usually the same. The wheat-Hessian fly interaction is similar to many plant-pathogen interactions in that it exhibits a gene-for-gene interaction (Hatchett and Gallun 1970). However, the regulation of primary metabolic genes responding to Hessian fly attack was quite different from that of plant genes responding to pathogen attack. During wheat-Hessian fly interaction, primary metabolic genes were regulated completely differently between plants during compatible interactions and plants during incompatible interactions (Fig. 2). The distinct regulation observed in the primary metabolic genes may also occur for genes with other functions, according to our microarray analysis (M.-S. Chen unpublished observation). As a result of distinct gene regulation in plants during compatible interactions and plants during incompatible interactions, the alteration in metabolite content was also different between plants during compatible and incompatible interactions. Among the 36 metabolites with significant changes in host plants following Hessian fly attack, only six (17\%) changed in the same direction (Table 2). Clearly, host response to Hessian fly attack was different from host response to pathogen attack in terms of gene regulation and metabolite accumulation. The different responses might indicate different molecular mechanisms linked with specific host-plant resistance or susceptibility in different plant-parasite interactions. The distinct gene regulation in wheat-Hessian fly interaction is consistent with distinct plant phenotypes between compatible and incompatible interactions. Plants during compatible interactions are completely and irreversibly stunted by Hessian fly attack, whereas plants during incompatible interactions grow normally after initial growth deficits (Anderson and Harris 2006).

\section{C/N shift and key metabolic pathways.}

There was a shift from $\mathrm{C}$-compounds to $\mathrm{N}$-compounds in plants during both compatible and incompatible interactions. However, the $\mathrm{C} / \mathrm{N}$ shift was much more dramatic in plants during compatible interactions than in plants during incompatible interactions (Table 1). Since N-compounds, such as amino acids, may provide better nutrition to insects, the dramatic $\mathrm{C} / \mathrm{N}$ shift in plants during compatible interactions might be a necessary condition for insect growth and development and, therefore, for host plant susceptibility (Harris et al. 2006; Schwachtje et al. 2006).

In plants during compatible interactions, there was a $36 \%$ decrease in C-compounds and a $46 \%$ increase in $\mathrm{N}$-compounds (Table 1). The shift from $\mathrm{C}$-compounds to $\mathrm{N}$-compounds was likely mediated through a coordinated regulation of primary metabolic pathways, such as glycolysis, the TCA cycle, and amino-acid synthesis. Assuming the protein levels and enzymatic activities are proportional to RNA levels, the overall increase in the transcripts of glycolytic genes may suggest that more sugars were catabolized via glycolysis. Consistent with this trend, the concentration of glucose, fructose, mannose,

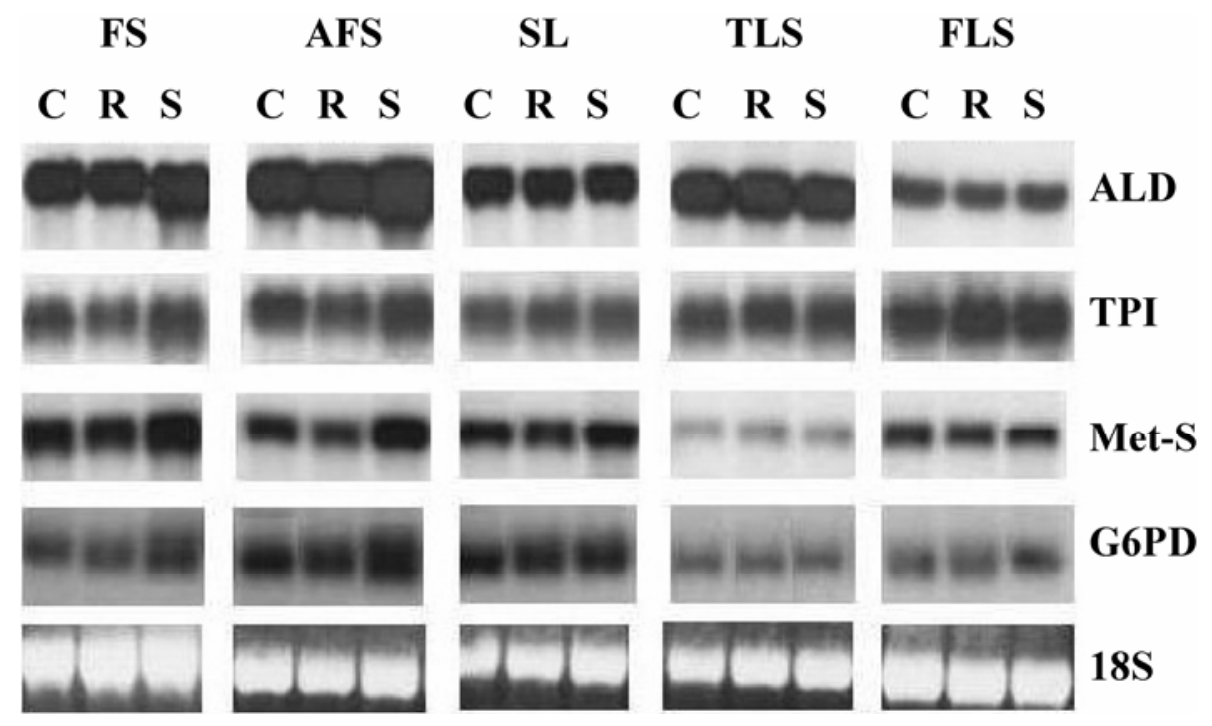

Fig. 3. Analysis of changes in gene transcripts in different tissues after Hessian fly attack. FS $=$ feeding site (second leaf-sheath towards the base), AFS $=$ immediately above feeding site (second leaf-sheath towards the leaf), $\mathrm{SL}=$ second leaf, TLS $=$ third leaf sheath, and FLS = first leaf sheath. C, R, and S = control plants, plants during incompatible interactions, and plants during compatible interactions, respectively. ALD $=$ fructose 1,6 -biphosphate aldolase, TPI $=$ triose phosphate isomerase, Met-S = methionine synthase, G6PD = glucose-6-phosphate dehydrogenase, and 18S = 18 S ribosomal RNA. 
glucose-6-phosphate, trehalose, ribulose-5-phosphate, and xylose decreased 49 to $85 \%$ (Table 2) in plants during compatible interactions. Glucose and glucose-6-phosphate are direct substrates of glycolysis, while other sugars can also enter into glycolysis after their conversion into fructose-6-phosphate or other intermediates.

Pyruvate, the final product of glycolysis, can be used either for amino-acid synthesis directly or converted into acetyl-CoA to enter the TCA cycle. In contrast to glycolysis, which was upregulated overall, the TCA cycle was up-regulated only at certain steps (Fig. 2). The unbalanced upregulation of the genes in the TCA cycle might suggest a net production of certain intermediates for biosynthesis, such as amino-acid synthesis, assuming RNA, protein, and enzymatic activity levels are correlated.

All of the amino-acid synthesis genes examined were upregulated significantly in plants during compatible interactions (Fig. 2). Again, if RNA, protein, and enzymatic activity levels are proportional, the upregulation of these genes might suggest that more amino acids were synthesized. Indeed, the levels of some amino acids were elevated dramatically, including proline $(1,249 \%)$, arginine or ornithine $(64 \%)$, serine $(60 \%)$, threonine (46\%), and aspartic acid (39\%) (Table 2). The increase in amino acids was comparable to the decrease in TCA cycle intermediates, including citric acid $(-49 \%), \alpha$-ketoglutaric acid $(-73 \%)$, fumarate $(-62 \%)$, and malate $(-61 \%)$. Together, this indicated that metabolic intermediates from glycolysis and the TCA cycle were used for amino-acid synthesis in plants during compatible interactions.

In addition to carbon intermediates, amino-acid synthesis also requires NADPH and nitrogen. NADPH can be produced through PPP. The increase in transcripts of G6PD suggests that PPP activity increased in plants during compatible interactions, since $G 6 P D$ is the rate-limiting enzyme in this pathway. The decrease in concentration of ribulose-5-phosphate $(-85 \%)$, a major substrate in PPP, indicated that ribulose-5-phospahte was rapidly used in biosynthesis. The need for nitrogen could be achieved through more nitrogen assimilation via the urea cycle. An increase in the activity of the urea cycle was suggested by the fact that the concentration of citrulline, arginine, and ornithine, the key components of the urea cycle, increased significantly (Table 2).

\section{The impact of the $\mathrm{C} / \mathrm{N}$ shift.}

The shift from $\mathrm{C}$-compounds to $\mathrm{N}$-compounds in plants during compatible interactions probably affects both the insect and the host plant. The impact on the insect is obvious-greater amounts of amino acids provide better nutrition for insect growth and development. Interestingly, the most dramatically increased amino acid was proline, which increased $1,249 \%$ in plants during compatible interactions. The large increase in proline suggested that a water-deficit stress might be associated with Hessian fly-wheat compatible interactions. Free proline usually increases in plants under drought conditions, and the increase in proline enhances plant drought tolerance (Yamada et al. 2005). Elevated concentrations of proline can also attract and stimulate insect feeding (Haglund 1980; Mattson and Haack 1987). Proline is also a very important energy reserve for insects including the tsetse fly Glossina morsitans (Bursell 1978) and Colorado potato beetle Leptinotarsa decemlineata (Brouwers and de Kort) 1979). However, there is no evidence that the concentration of proline affects host-plant susceptibility to insects (Bright et al. 1982). Further research will have to be conducted to determine the biological significance of the large elevation in proline concentration during the wheat-Hessian fly compatible interaction.

The $\mathrm{C} / \mathrm{N}$ shift will impact the plant as well. The decrease in $\mathrm{C}$-compounds such as glucose and xylose may inhibit the growth and development of plants because these compounds are necessary substrates for the biosynthesis of cellular polymers, such as DNA and cell wall components. Indeed, in the case of the wheat-Hessian fly interaction, the growth of wheat seedlings during compatible interactions is completely inhibited (Anderson and Harris 2006). In summary, the dramatic $\mathrm{C} / \mathrm{N}$ shift in plants during compatible interactions may be part of the mechanisms for the inhibition of wheat growth while creating a favorable environment for insect growth and development (Bronner 1992; Harris et al. 2006).

\section{The regulation of the $\mathrm{C} / \mathrm{N}$ shift.}

How might the shift from $\mathrm{C}$-compounds to $\mathrm{N}$-compounds in plants during compatible interactions be regulated? For many metabolic pathways, including glycolysis, the TCA cycle, and amino-acid synthesis, there is a feedback mechanism for gene regulation (Plaxton 1996). This feedback may be one of the regulatory mechanisms for the $\mathrm{C} / \mathrm{N}$ shift. First, Hessian fly feeding results in removal of small soluble nutrients, such as free sugars and amino acids. The decrease in content of free sugars might then be compensated by the transport of photoassimilates from other parts of the plants to the feeding site. The amino acids removed by insect feeding, however, will have to be compensated by more biosynthesis, since degradation of proteins cannot be sustained as the insect continues to feed. The synthesis of more amino acids results in the decrease of metabolic intermediates such as NADPH, $\alpha$-ketoglutarate, pyruvate, and 3-phosphoglycerate. The decrease in these intermediates may then activate the transcription of related genes via the feedback mechanism. The coordinated activation of aminoacid synthesis pathways, glycolysis, and TCA cycle may have resulted in a shift from $\mathrm{C}$-compounds into $\mathrm{N}$-compounds, providing that these changes are also reflected in protein and enzymatic levels.

While evidence points to feedback regulation, active control mechanisms were also in operation. For example, the prolonged increase in the transcripts of the P5CS gene (involved in proline synthesis; Fig. 2D) and the dramatic increase in proline concentration suggested the existence of one or more active regulatory mechanisms. However, how the pathways are actively regulated remains unknown.

\section{MATERIALS AND METHODS}

\section{Plants and insects.}

Wheat (Triticum aestivum) genotype 'Molly' and two Hessian fly populations, biotype $G P$ and biotype $v H 13$, were used in this study. 'Molly' carries the Hessian fly resistance gene $H 13$, which is compatible to biotype $v H 13$ but incompatible to biotype GP (Patterson et al. 1994; Rider et al. 2002). All Hessian fly populations were maintained on two-leaf seedlings of the compatible wheat cultivar Karl 92 in environmental chambers at $20^{\circ} \mathrm{C}$ and 12 -h-light and12-h-dark photoperiod. The insect can be stored as puparium at $4^{\circ} \mathrm{C}$ for over a year.

\section{Experimental treatment and design.}

Three treatments were included in the experiment, including control, compatible interaction, and incompatible interactions. Controls were noninfested 'Molly' plants; compatible interactions were 'Molly' plants infested with virulent insects, biotype $v H 13$ Hessian flies, whereas incompatible interactions were 'Molly' plants infested with avirulent insects, biotype GP Hessian flies. All treatments were arranged as randomized complete block design in growth chambers. Each treatment was repeated two times (with independent infestations) for Northern blot analysis and six times for metabolic profiling. For Northern blots, each sample was derived from pooled tissues of five 
plants. For metabolic profiling, each sample was prepared from pooled tissues of 20 plants.

\section{Sample collection.}

'Molly' seeds (15 to 30) were planted in each pot in a growth chamber programmed at 20 and $18^{\circ} \mathrm{C}$ in a 14-h-light and 10-h-dark cycle. At the 1.5-leaf stage, one mated female (with ovipositor retracted) per plant of biotypes GP or $v H 13$, confined in a mesh cage, were released onto plants. Control plants were grown under the same conditions but were not exposed to egg-laying females. Under these conditions, first instar larvae crawled down to the base of the wheat plant and attacked epidermal cells on the abaxial side of the sheath of the second leaf. To sample the attack (feeding) site, we collected the basal 10-mm long section of the leaf sheath where larvae feed. For samples above the feeding site, we removed the $10-\mathrm{mm}$ section of leaf sheath adjacent to the feeding site section. To determine tissue-specific gene expression, leafsheath samples were harvested $72 \mathrm{~h}$ after initial larval attack. Samples were also collected from the second leaf blade, the third leaf sheath, and the first leaf sheath for comparison. For RNA extraction, samples were collected at the feeding site 12, $24,48,72,120$, and $168 \mathrm{~h}$ after initial larval attack. For metabolic profiling, samples were collected from Hessian fly attack sites $72 \mathrm{~h}$ after initial larval attack.

\section{RNA isolation.}

Total RNA was extracted from plant tissue using TRI reagent (Molecular Research Center Inc, Cincinnati, OH, U.S.A.), following the protocol provided by the manufacturer. Equal amounts (approximately $5 \mu \mathrm{g}$ ) of total RNA of each sample were separated on $1.5 \%$ agarose gels containing formaldehyde, were blotted onto a GeneScreen membrane (Perkin Elmer Life Science Inc., Boston), and were baked at $80^{\circ} \mathrm{C}$ for $2 \mathrm{~h}$ after the transfer.

\section{Northern hybridization.}

Two types of probes were used for Northern blot analysis. Oligonucleotide probes were designed from cDNA sequences in the National Center for Biotechnology Information database. cDNA probes were made from polymerase chain reaction fragments amplified using specific primers designed according to wheat expressed sequence tag sequences or the conserved regions of genes from rice, maize, and barley, if the wheat counterparts were not available. The accession numbers, names, and functions or putative functions of the genes represented by these sequences, EC numbers of the encoding enzymes, and their metabolic pathways are listed in Table 3. cDNA probes were labeled with ${ }^{32} \mathrm{P}$-dCTP using the random labeling kit from Stratagene (La Jolla, CA, U.S.A.), while oligonucleotide probes were labeled with $\gamma-{ }^{32} \mathrm{P}$-ATP using T4 polynucleotide kinase (Invitrogen, Carlsbad, CA, U.S.A.). Hybridization, washing, and image analysis were carried out as described previously (Chen et al. 2004).

\section{Metabolic profiling.}

Gas chromatographic-mass spectrometry (GC-MS) metabolic profiling was performed in a way to measure free metabolites and was carried out in the Metabolomics Core Lab at the University of California Davis via a commercial contract. Briefly, samples were processed through three steps: solvent extraction, sample derivatization, and GC-MS analysis. For solvent extraction, $0.25 \mathrm{ml}$ of precooled $\left(-20^{\circ} \mathrm{C}\right)$ solvent containing water, acetonitrile, and isopropanol $(2: 3: 3, \mathrm{vol} / \mathrm{vol} / \mathrm{vol})$ was added to a sample consisting of $5 \mathrm{mg}$ of frozen ground wheat tissues. Samples were agitated for $5 \mathrm{~min}$ at $4^{\circ} \mathrm{C}$ on a chilling and heating dry bath. Following the extraction, samples were centrifuged for $2 \mathrm{~min}$ at $13,200 \mathrm{rpm}$, and $0.5 \mathrm{ml}$ of the supernatant was transferred to a clean tube. The samples were dried via speed vacuum. For sample derivation, $20 \mu \mathrm{l}$ of a methoxylamine hydrochloride in pyridine mixture $(40 \mathrm{mg} / \mathrm{ml})$ was added to each sample. The mixture was agitated on a dry bath for 30 min at $40^{\circ} \mathrm{C}$, followed by addition of $180 \mu \mathrm{l}$ of $N$-methyltrimethylsilylacetamide (MSTFA). Samples were then heated at $40^{\circ} \mathrm{C}$ for $30 \mathrm{~min}$ in an orbital. GC-MS was performed using a $6890 \mathrm{~N}$ Agilent (Atlanta) gas chromatograph interfaced to a time of flight Pegasus III mass spectrometer (Leco Corp., St. Joseph, MI, U.S.A.). The devices were equipped with automated injection and cooling systems. Chromatography was performed on a $30 \mathrm{~m} \times 0.25 \mathrm{~mm}$ Rtx-5Sil MS column with a $0.25-\mu \mathrm{m}$ integra guard column (Restek, Bellefonte, PA, U.S.A.).

\section{Data normalization and statistical analysis.}

The data (peak area of each individual metabolite) were first normalized by dividing the peak area of each individual metabolite with the total peak area in each sample. The normalized data were subjected to statistical analysis using the SAS procedure. Multiple comparisons of the means of different treatments were computed using Fisher's least significant difference (LSD) (SAS Institute, Inc. Cary, NC, U.S.A.). The data

Table 3. Probe information

\begin{tabular}{|c|c|c|c|c|}
\hline Accession no. & Name/function of gene & Abbreviation & EC no. & Metabolic pathway \\
\hline AJ420778 & Fructose 1-,6-biphosphate aldolase & ALD & EC 4.1.2.13 & Glycolysiss \\
\hline CA763752 & Cytosolic triose phosphate isomerase & TPI & EC 5.3.1.1 & Glycolysis \\
\hline AF273738 & Cytosolic glyceraldehyde-3-phosphate dehydrogenase-like gene & G3PDH & EC 1.2.1.9 & Glycolysis \\
\hline X15232 & Cytosolic phosphoglycerate kinase & PGK & EC 2.7.2.3 & Glycolysis \\
\hline EF210576 & A putative cofactor-independent phosphoglycerate mutase & & EC 5.4.2.1 & Glycolysis \\
\hline AY335488 & Enolase & ENOL & EC 4.2.1.11 & Glycolysis \\
\hline AY623894 & Cytosolic pyruvate kinase & PK & EC 2.7.1.40 & Glycolysis \\
\hline AY236152 & Cytosolic malate dehydrogenase & MD & EC 1.1.1.37 & TCA \\
\hline AY775790 & $\mathrm{NAD}^{+}$dependent isocitrate dehydrogenase & ICD & EC 1.1.1.41 & TCA \\
\hline AF475119 & Succinate dehydrogenase & SD & EC 1.3.5.1 & TCA \\
\hline CK207322 & $\mathrm{NAD}^{+}$-dependent malic enzyme & ME & EC 1.1.1.40 & TCA \\
\hline CD886657 & $\alpha$-Ketoglutarate dehydrogenase ( 2 -oxoglutarate dehydrogenase, E1 subunit) & OD & EC 1.2.4.2 & TCA \\
\hline AJ007705 & Phosphoenolpyruvate carboxylase & PEPC & EC 4.1.1.31 & TCA \\
\hline AB029456 & Glucose-6-phosphate dehydrogenase & G6PD & EC 1.1.1.49 & PPP \\
\hline DQ124211 & Glutamine synthetase & Gln-S & EC 6.3.1.2 & Gln synthesis \\
\hline AB008845 & NADH-dependent glutamate synthase & Glu-S & EC 1.4.1.14 & Glu synthesis \\
\hline AF022914 & Pyrroline-5-carboxylate synthetase & P5CS & $\mathrm{EC} 1.2 .1 .41$ & \\
\hline EC 2.7.2.11 & Pro synthesis & & & \\
\hline AF439723 & Methionine synthase & Met-S & EC 2.1.1.13 & Met synthesis \\
\hline
\end{tabular}

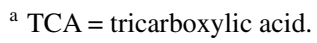


sets with LSD significance were reanalyzed through a Dunnett's test to avoid a type I error (false positive). Both LSD and Dunnett tests showed the same significant levels in our data sets. Principal component analysis was performed using the software package SYSTAT, version 10 (Systat Software Inc., Richmond, CA, U.S.A.).

\section{ACKNOWLEDGMENTS}

The authors thank V. Tolstikov in the Metabolomics Core Lab at the University of California Davis for the measurements of metabolites and for providing the written measurement procedures, R. Welti for providing critical reading, and J. Marshall, J. Bai, and J. Shah for reviewing an earlier version of the manuscript. This paper is contribution number 07-163-J from the Kansas Agricultural Experiment Station. Hessian fly voucher specimens (No. 150) are located in the KSU Museum of Entomological and Prairie Arthropod Research, Kansas State University, Manhattan, KS, U.S.A. This research was supported by two grants from the National Research Initiative Competitive Grant program, the United States Department of Agriculture (USDA 04-35607-14861, and USDA2005-35302-16254).

\section{LITERATURE CITED}

Agrios, G. N. 1997. Plant diseases caused by Mollicutes: Phytoplasmas and spiroplasmas. Pages 457-470 in: Plant Pathology, 4th ed. G. N. Agrios, ed. Academic Press, New York.

Anderson, K. G., and Harris, M. O. 2006. Does R gene resistance allow wheat to prevent plant growth effects associated with Hessian fly (Diptera: Cecidomyiidae) attack? J. Econ. Entomol. 99:1842-1853.

Berzonsky, W. A., Ding, H., Haley, S. D., Lamb, R. J., McKenzie, R. I. H., Ohm, H. W., Patterson, F. L., Peairs, F. B., Porter, D. R., Ratcliffe, R. H., and Shanower, T. G. 2003. Breeding wheat for resistance to insects. Plant Breed. Rev. 22:221-296.

Bright, S. W. J., Lea, P. J., Kueh, J. S. H., Woodcock, C., Hollomon, D. W., and Scott, G. C. 1982. Proline content does not influence pest and disease susceptibility of barley. Nature 295:592-593.

Bronner, R. 1992. The role of nutritive cells in the nutrition of cynipids and cecidomyiids. Pages 118-140 in: Biology of Insect-Induced Galls. J. D. Shorthouse and D. Rohfritsch, eds. Oxford University Press, New York.

Brouwers, E. V. M., and de Kort, C. A. D. 1979. Amino acid metabolism during flight in the Colorado potato beetle, Leptinotarsa decemlineata. J. Insect Physiol. 25:411-414.

Bursell, E. 1978. Quantitative aspects of proline utilization during flight in tsetse flies. Physiol. Entomol. 3:265-272.

Chen, M. S., Fellers, J. P., Stuart, J. J., Reese, J. C. and Liu, X. 2004. A group of related cDNAs encoding secreted proteins from Hessian fly [Mayetiola destructor (Say)] salivary glands. Insect Mol. Biol. 13:101108.

Dangl, J. L., and McDowell, J. M. 2006. Two modes of pathogen recognition by plants. Proc. Natl. Acad. Sci. U.S.A. 103:8575-8576.

Dodds, P. N., Lawrence, G. J., Catanzariti, A.-M., Teh, T., Wang, C.-I. A., Ayliffe, M. A., Kobe, B., and Ellis, J. G. 2006. Direct protein interaction underlies gene-for-gene specificity and coevolution of the flax resistance genes and flax rust avirulence genes. Proc. Natl. Acad. Sci. U.S.A. 103:8888-8893.

Ferry, N., Edwards, M. G., Gatehouse, J. A., and Gatehouse, A. M. R. 2004. Plant-insect interactions: Molecular approaches to insect resistance. Curr. Opin. Biotechnol. 15:155-161.

Gagne, R. J., and Hatchett, J. H. 1989. Instars of the Hessian fly (Diptera: Cecidomyiidae). Ann. Entomol. Soc. Am. 82:73-79.

Girousse, C., Moulia, B., Silk, W., and Bonnemain, J. L. 2005 Aphid infestation causes different changes in carbon and nitrogen allocation in alfalfa stems as well as different inhibitions of longitudinal and radial expansion. Plant Physiol. 137:1474-1484.

Goethals, K., Vereecke, D., Jaziri, M., Van Montagu, M., and Holsters, M. 2001. Leafy gall formation by Rhodococcus fascians. Annu. Rev. Phytopathol. 39:27-52.

Haglund, B. M. 1980. Proline and valine-cues which stimulate grasshopper herbivory during drought stress? Nature 288:697-698.

Hahlbrock, K., Bednarek, P., Ciolkowski, I., Hamberger, B., Heise, A., Liedgens, H., Logemann, E., Nürnberger T., Schmelzer, E., Somssich, I. E., and Tan, J. 2003. Non-self recognition, transcriptional reprogramming, and secondary metabolite accumulation during plant/ pathogen interactions. Proc. Natl. Acad. Sci. U.S.A. 100:14569-14576.
Harris, M. O., Stuart, J. J., Mohan, M., Nair, S., Lamb, R. J., and Rohfritsch, O. 2003. Grasses and gall midges: Plant defense and insect adaptation. Annu. Rev. Entomol. 48:549-577.

Harris, M. O., Freeman, T. P., Rohfritsch, O., Anderson, K. G., Payne, S. A., and Moore, J. A. 2006. Virulent Hessian fly (Diptera: Cecidomyiidae) larvae induce a nutritive tissue during compatible interactions with wheat. Ann. Entomol. Soc. Am. 99:305-316.

Hatchett, J. H., and Gallun, R. L. 1970. Genetics of the ability of the Hessian fly, Mayetiola destructor, to survive on wheat having different genes for resistance. Ann. Entomol. Soc. Am. 63:1400-1407.

Kaloshian, I., 2004. Gene-for-gene disease resistance: Bridging insect pest and pathogen defense. J. Chem. Ecol. 30:2419-2438.

Kessler, A., and Baldwin, I. T. 2002. Plant responses to insect herbivory. Annu. Rev. Plant Biol. 53:299-328.

Liu, X. M., Gill, B. S., and Chen, M. S. 2005. Hessian fly resistance gene $H 13$ is mapped to a distal cluster of $R$ genes in chromosome 6DS of wheat. Theor. Appl. Genet. 111:243-249

Maleck, K., Levine, A., Eulgem, T., Morgan, A., Schmid, J., Lawton, K. A., Dangl, J. L., and Dietrich, R. A. 2000. The transcriptome of Arabidopsis thaliana during systemic acquired resistance. Nat. Genet. 26:403-410.

Mattson, W. J., and Haack, R. A. 1987. The role of drought in outbreaks of plant-eating insects. BioScience 37:110-118.

Meyers, B. C., Dickerman, A. W., Michelmore, R. W., Pecherer, R. M., Sivaramakrishnan, S., Sobral, B. W., and Young, N. D. 1999. Plant disease resistance genes encode members of an ancient and diverse protein family within the nucleotide-binding superfamily. Plant J. 20:317-332.

Nimchuk, Z., Eulgem, T., Holt, B. F. III, and Dangl, J. L., 2003. Recognition and response in the plant immune system. Annu. Rev. Genet. 37:579-609.

Patterson, F. L., Mass, F. B., III, Foster, J. E., Ratcliffe, R. H., Cambron, S., Safanski, G., Taylor, P. L., and Ohm, H. W. 1994. Registration of eight Hessian fly resistant common winter wheat germplasm lines (Carol, Erin, Flynn, Iris, Joy, Karen, Lola, and Molly). Crop Sci. 34:315-316.

Plaxton, W. C. 1996. The organization and regulation of plant glycolysis Annu. Rev. Plant Physiol. Plant Mol. Biol. 47:185-214.

Rohfritsch, O. 2005. Gall making. Pages 1021-1022R in: Goodman Encyclopedia of Plant and Crop Science. Marcel Dekker, New York.

Rider, S. D., Jr., Sun, W., Ratcliffe, R. H., and Stuart, J. J. 2002. Chromosome landing near avirulence gene vH13 in the Hessian fly. Genome 45:812-822.

Schwachtje, J., Minchin, P. E. H., Jahnke, S., van Dongen, J. T., Schittko, U., and Baldwin, I. T. 2006. SNF1-related kinases allow plants to tolerate herbivory by allocating carbon to roots. Proc. Natl. Acad. Sci. U.S.A. 103:12935-12940.

Shukle, R. H., Grover, P. B., and Foster, J. E. 1990. Feeding of Hessian fly (Diptera: Cecidomyiidae) larvae on resistant and susceptible wheat. Environ. Entomol. 19:494-500.

Stone, G. N., and Schönrogge, K. 2003. The adaptive significance of insect gall morphology. Trends Ecol. Evol. 18:512-522.

Tang, X., Frederick, R. D., Zhou, J., Halterman, D. A., Jia Y., and Martin, G. B. 1996. Initiation of plant disease resistance by physical interaction of Avrpto and Pto kinase. Science 274:2060-2063.

Tao, Y., Xie, Z., Chen, W., Glazebrook, J., Chang, H. S., Han, B., Zhu, T., Zou, G., and Katagiri, F. 2003. Quantitative nature of Arabidopsis responses during compatible and incompatible interactions with the bacterial pathogen Pseudomonas syringae. Plant Cell 15:317-330.

Walling, L. L. 2000. The myriad plant responses to herbivores. J. Plant Growth Reg. 19:195-216.

Wan, J., Duning, F. M., and Bent, A. F. 2002. Probing plant-pathogen interactions and downstream defense signaling using DNA microarrays. Funct. Integr. Genomics 2:259-273.

Yamada, M., Morishita, H., Urano, K., Shiozaki, N., Yamaguchi-Shinozaki, K., Shinozaki, K., and Yoshiba, Y., 2005. Effects of free proline accumulation in petunias under drought stress. J. Exp. Bot. 56:1975-1981.

Zupan, J., Muth, T. R., Draper, O., and Zambryski, P. 2000. The transfer of DNA from Agrobacterium tumefaciens into plants: A feast of fundamental insights. Plant J. 23:11-28.

\section{AUTHOR-RECOMMENDED INTERNET RESOURCE}

University of California Davis Metabolomics Core identified compounds webpage: metacore.ucdavis.edu/techno1/compounds

National Center for Biotechnology Information database: www.ncbi.nlm.nih.gov 